\section{Rapid Localization of a Gene within BACs and PACs}

\section{BioTechniques 32:240-242 (February 2002)}

The generation of transgenic animals using a promoter to direct the expression of a gene of interest is a continuously developing technique. Traditionally, a short fragment from the promoter of a gene with the desired expression profile (plasmid-based transgene) has been used as a vector to express a gene of interest. However, more recently, large pieces of DNA such as yeast artificial chromosomes (YACs), bacterial artificial chromosomes (BACs), and P1-based artificial chromosomes (PACs) are replacing plasmid-based transgenes, which is due mainly to three reasons: $(i)$ large pieces of DNA are more likely to contain all of the promoter elements needed for faithful gene expression; (ii) expression of the gene of interest is more likely to be copy number dependent; and (iii) there is less variability in the pattern of expression across transgenic lines $(2,3)$. Recently, the modification of BACs and PACs has become a routine technique $(5,6)$. Usually, the gene of interest is placed downstream of the ATG of a chosen gene in the BAC or PAC. The rationale for using $\mathrm{BACs}$ and PACs is that these large fragments of DNA are likely to contain all or most of the cis elements that regulate the expression of the gene chosen to drive the expression of the transgene. Therefore, it is of obvious importance to know the localization of the gene chosen for expression within the BAC or PAC, as a localization too proximal to one of the ends can result in a loss of some regulatory elements upstream or downstream of the ATG needed to drive faithful gene expression. This orientation can be achieved by restriction analysis, which is usually difficult and cumbersome. Here, we present a straightforward method that allows simultaneous modification of a BAC or PAC and the localization of the gene within the BAC or PAC in a single step.

For this purpose, we generated an expression cassette containing the follow- ing features: the gene to be expressed (here the iCre recombinase), a codonimproved Cre recombinase (4) with a polyadenylation signal from bovine growth hormone (BD Biosciences Clontech, Palo Alto, CA, USA) and an ampicillin resistance cassette flanked by two Flp recombinase target (FRT) sites and containing an internal Not I restriction site to allow the localization of the gene within the PAC or BAC (Figure 1).

A $120-\mathrm{kb}$ PAC containing the Kainic receptor 1 gene $\left(\mathrm{KA}_{1}\right)$ was isolated for use as a vector to drive the expression of the iCre recombinase. The $\mathrm{KA}_{1} \mathrm{PAC}$ was modified by ET-recombination (homologous recombination in E. coli) as previously described (6). Briefly, a 150-bp homology arm to the $\mathrm{KA}_{1}$ gene was amplified by PCR and cloned in front of the iCre recombinase, replacing the internal ATG of the $\mathrm{KA}_{1}$ receptor gene with the iCre ATG. A 3' homology region to the $\mathrm{KA}_{1}$ gene of $150 \mathrm{bp}$ was amplified by PCR and cloned at the end of the construct (Figure 1). DH10B bacteria containing the $\mathrm{KA}_{1}$ PAC were transformed with a tetracycline-resistant version of the plasmid pBAD ET $\gamma$ [plasmid that contains the necessary elements to carry out homologous recombination and modification of the PAC (6)]. The cells were induced with arabinose $(0.2 \%)$, and competent cells were generated. The digested targeting vector was electroporated into competent cells previously induced with arabinose and containing the $\mathrm{KA}_{1}$ PAC. Putative positive colonies believed to have undergone homologous recombination were selected in the presence of kanamycin (PAC vector resistance) and ampicillin. Homologous recombination of the targeting construct with the appropriate sequences in the PAC was confirmed by restriction analyses, Southern analyses, and PAC sequencing. The frequency of correct targeted PACs was 8 of 10 (i.e., 8 of 10 colonies that had undergone recombination had the iCre cassette inserted in the right place).

The localization of the $\mathrm{KA}_{1}$ gene within the PAC was performed as follows. The modified PAC KA $\mathrm{A}_{1}$ was subjected to Not I digestion and analyzed by pulsed-field gel electrophoresis (Figures 1 and 2). The size-separated DNA was transferred to nylon membrane and probed with a radiolabeled oligonucleotide immediately upstream

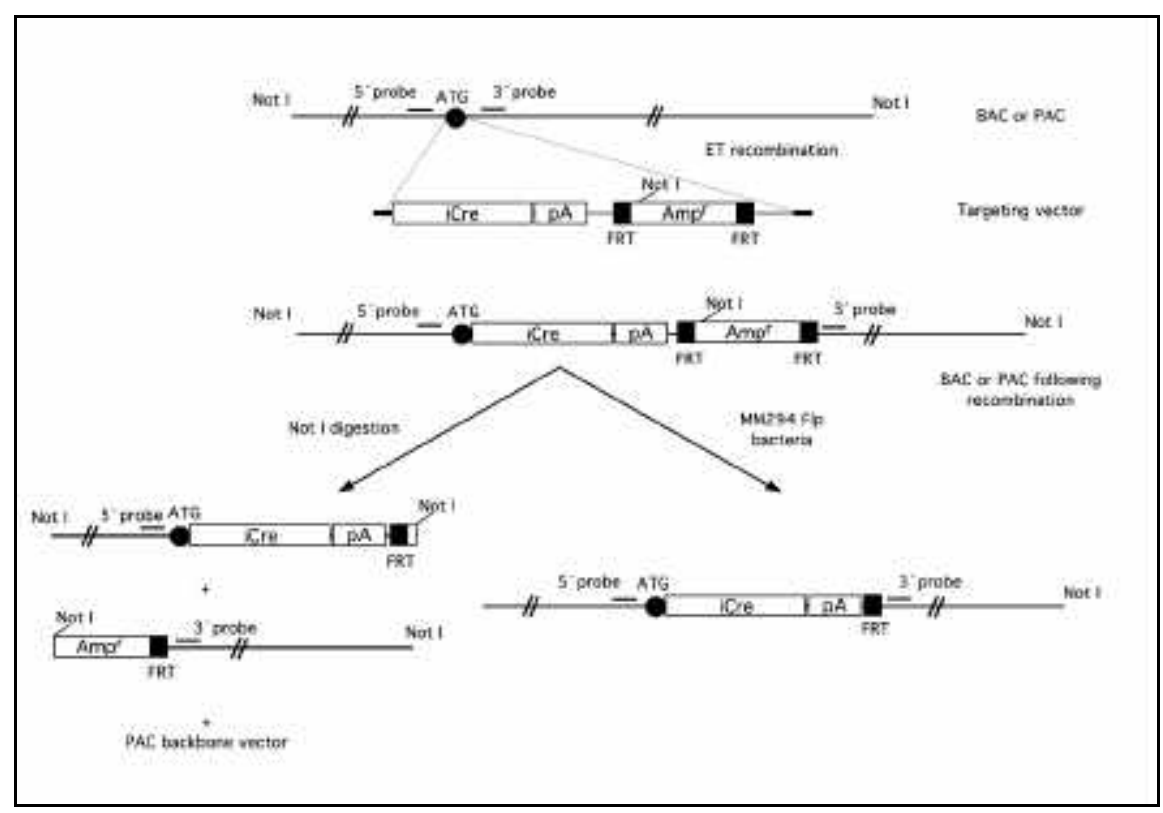

Figure 1. Schematic representation of the modification of the KA PAC. Shown is an expression cassette containing the iCre, a growth hormone polyadenylation signal, and an ampicillin resistance cassette flanked by two FRT sites and containing an internal NotI recognition site. The cassette was recombined into the $\mathrm{KA}_{1} \mathrm{PAC}$, and the location of the $\mathrm{KA}$ gene within the PAC was determined by a NotI digestion. The probes used to identify the $5^{\prime}$ and $3^{\prime}$ regions from the endogenous KA ATG are denoted $5^{\prime}$ probe and $3^{\prime}$ probe, respectively. The ampicillin cassette and internal NotI site are removed upon electroporation of the modified PAC DNA into MM294 Flp-expressing bacteria. 
of the ATG of the $\mathrm{KA}_{1}$ gene ( $5^{\prime}$ probe), which detected a fragment of $90 \mathrm{~kb}$. Following stripping, the membrane was hybridized with a 3 ' probe (immediately downstream of the ATG of the KA gene), which detected a $40-\mathrm{kb}$ fragment. From these analyses, we estimated that the $\mathrm{KA}_{1}$ gene was contained within the last third of the PAC with a 90-kb $5^{\prime}$ and a 40-kb $3^{\prime}$ sequence. A third fragment of $17 \mathrm{~kb}$ was present in the pulsed-field electrophoresis gel and corresponded to the backbone of the PAC vector following Not I digestion.

Following homologous recombination of the targeting vector in bacteria, the ampicillin cassette is no longer necessary and could interfere with the expression of the desired gene. Therefore, it is preferable to remove it. Based on this fact, the NotI site was placed between the FRT sites flanking the ampicillin cassette, allowing its removal upon treatment with Flp recombinase. The removal of the internal NotI site in this way has the added advantage that the modified PAC insert can again be excised intact from its vector backbone using a Not I digestion for subsequent oocyte injection. The modified $\mathrm{KA}_{1}$ PAC was electroporated in MM294 Flp-expressing bacteria (1) to remove the ampicillin cassette and the internal NotI site (Figures 1 and 2).

The use of large pieces of DNA such as BACs and PACs to drive expression of a transgene is becoming increasingly popular because of techniques that allow easy manipulation of the DNA and because the resulting transgene usually directs faithful expression. However, to take full advantage of BACs and PACs as expression vectors, the localization of the chosen gene within the BAC or PAC is required. Here, we present a strategy that allows the straightforward localization of a desired gene within a $\mathrm{BAC}$ or PAC in a simple and straightforward way. The whole process comprises ( $i$ ) PCR amplification and cloning of the homology arms flanking the iCre cassette, (ii) modification of the BAC/PAC using ET-cloning, (iii) orientation of the BAC/PAC combining a NotI digestion and Southern analysis using $5^{\prime}$ upspream and $3^{\prime}$ donwnstream probes, and (iv) removal of the ampicillin cassette flanked by two FRT sites. The combination of the modification of a BAC or PAC by ET-recombination (6) and the simultaneous introduction of a NotI recognition site (removed together with the prokaryotic selection cassette before oocyte injection of the transgene) will simplify the localization of a desired gene within a BAC or $\mathrm{PAC}$ in one unique step.

The simplicity of this method should allow fast and easy orientation of a gene into a PAC or PAC in comparison with the traditional time- and effort-demanding methods such as restriction analyses. To our knowledge, this is the first report in which, by a simple addition of a NotI site in the targeting construct used to modify a BAC or PAC, the position of a gene into a $\mathrm{BAC}$ or PAC can be easily determined.

\section{REFERENCES}

1.Buchholz, F., P.O. Angrand, and A.F. Stew art. 1996. A simple assay to determine the functionality of Cre or FLP recombination targets in genomic manipulation constructs. Nucleic Acids Res. 24:3118-3119.

2. Giraldo, P. and L. Montoliu. 2001. Size matters: use of YACs, BACs and PACs in transgenic animals. Transgenic Res. 10:83-103.

3.Schedl, A., L. Montoliu, G. Kelsey, and G. Schütz. 1993. A yeast artificial chromosome covering the tyrosinase gene confers copy number-dependent expression in transgenic mice. Nature 362:258-261.

4.Shimshek, D.R., J. Kim, D.J. Spergel, F. Buchholz, E. Casanova, A.F. Stewart, P.H. Seeburg, and R. Sprengel. Codon-improved Cre recombinase (iCre) expression in the mouse. Genesis (In Press.)

5. Yang, X.W., P. Model, and N. Heintz. 1997. Homologous recombination based modification in Escherichia coli and germline transmission in transgenic mice of a bacterial artificial chromosome. Nat. Biotechnol. 15:859-865.

6.Zhang, Y., F. Buchholz, J.P. Muyrers, and A.F.Stewart. 1998. A new logic for DNA engineering using recombination in Escherichia coli. Nat. Genet. 20:123-128.

2. Localization of the KA gene within the PAC.(A) A pulsed-field electrophoresis gel. Lane unmodified KA PAC digested with NotI. The upper band in this lane corresponds to the KA PAC insert $(120 \mathrm{~kb})$, and the lower band corresponds to the PAC vector backbone. Lane 2, modified KA PAC containing the iCre cassette and the introduced NotI site, digested with NotI. Digestion results in two fragments of 40 and $90 \mathrm{~kb}$ that correspond to sequences upstream and downstream of the internal KA gene ATG, respectively. Lane 3, modified KA PAC following electroporation into MM294 Flp-expressing bacteria digested with NotI. The ampicillin cassette and internal NotI have been removed. (B) Southern analyses of the gel described in panel A. The resulting blot was probed with a radiolabeled oligonucleotide upstream of the ATG ( $5^{\prime}$ probe), which hybridized with the $90-\mathrm{kb}$ fragment, stripped and probed with a radiolabeled oligonucleotide downstream of the ATG (3' probe), which detected the 40-kb fragment.
E.C. and T.L. contributed equally to this work. We thank Drs. B. Stride and W. Schmid for correcting this manuscript and helpful discussions. Address correspondence to Dr. Gunther Schütz, Abteilung 
Molekularbiologie der Zelle I., Deutsches Krebsforschungszentrum (DKFZ), Im Neuenheimer Feld 280, D-69120, Heidelberg Germany. e-mail: g.schuetz@dkfzheidelberg.de

Received 27 July 2001; accepted 29 October 2001.

E. Casanova, T. Lemberger, S. Fehsenfeld, E. Greiner, A.F. Stewart ${ }^{1}$, and G. Schütz Deutsches

Krebsforschungszentrum (DKFZ)

${ }^{1}$ European Molecular

Biology Laboratory (EMBL) Heidelberg, Germany

\section{One-Step RT-PCR for Screening Microdissected Tissue Samples}

BioTechniques 32:242-248 (February 2002)

The technology of laser-capture microdissection (LCM) enables the easy extraction of single cells or defined groups of cells from a tissue section $(1,2)$. This technique uses a laser pulse to activate a plastic polymer, which binds to and captures cells from a frozen or paraffin-embedded tissue section. Captured cells present on this solid support can then be used for nucleic acid and/or protein analysis. This technology is becoming increasingly important for the characterization of molecular profiles of cell subpopulations within a heterogeneous tissue (4).

Despite the enormous power of LCM technology, a constant challenge for investigators has been the consistent manipulation of small quantities of material. Most protocols require an amplification step, preceded by multistep procedures to isolate and purify the molecular components (3). For example, the steps involved in gene expression studies using RT-PCR include microdissection, denaturation of RNA using guanidinium isothiocyanatebased reagents, phenol-chloroform treatment to remove proteins, precipitation of nucleic acids, washing, resuspension of RNA, re-extraction of RNA when required for increased purity, reverse transcription to produce cDNA, and PCR using gene-specific primers. Although this standard protocol is effective, RNA can potentially be lost at each step.

In this report, we describe a one-step protocol for screening gene expression in microdissected cells using RT-PCR. Significantly, this procedure does not require an initial isolation of RNA. Rather, the reverse transcription and subsequent PCR amplification of specific genes are conducted directly from the microdissected cells. This procedure is a rapid, specific, and cost-effective screening technique.

When we started this project, we hypothesized that, because of the small

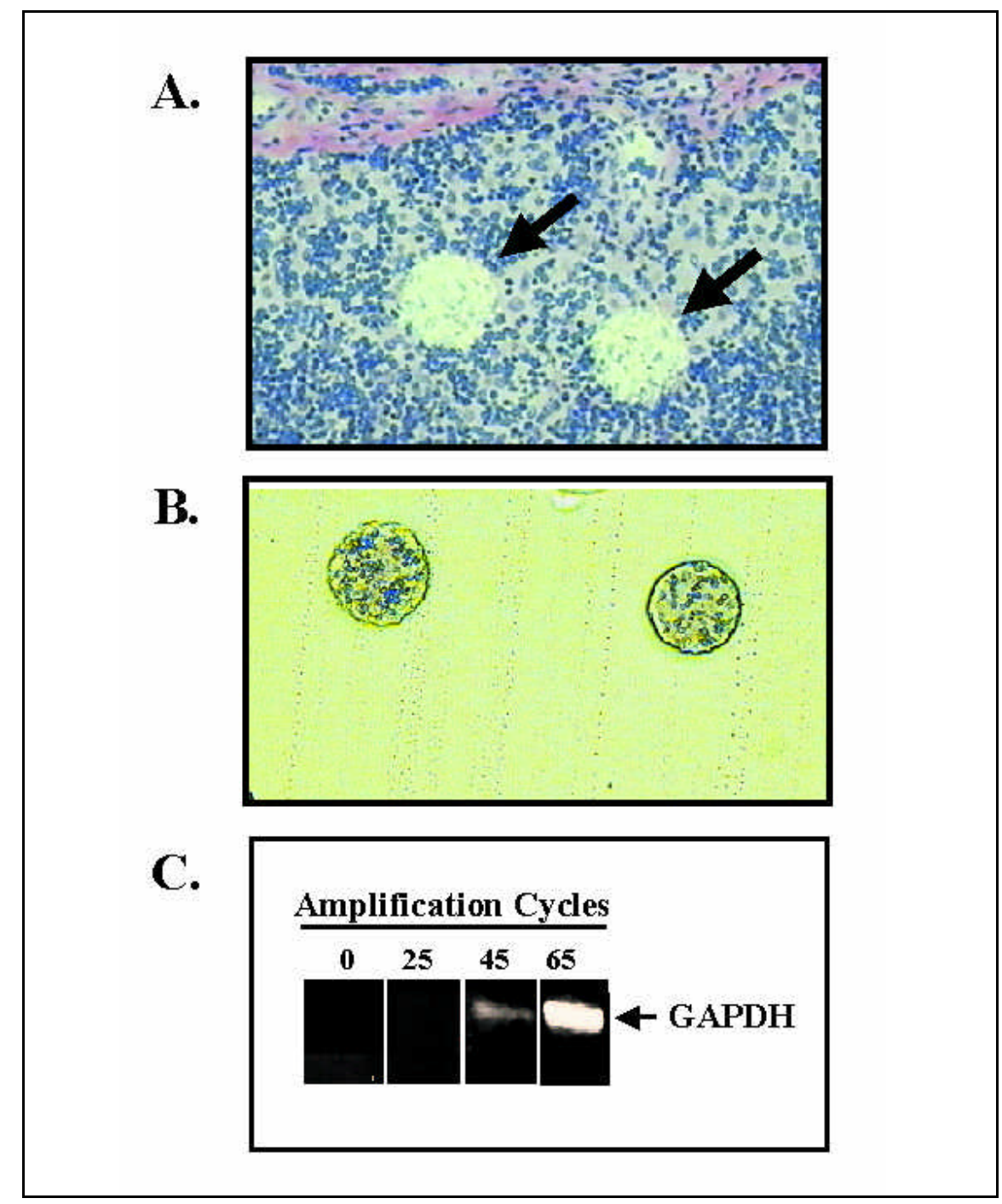

Figure 1. Microdissection and RT-PCR of cells from human remnant tonsil. In this representative image, the cells were microdissected from a frozen section of human tonsil, and the GAPDH was amplified using the one-step RT-PCR protocol. (A) Tonsil section from which two areas have been microdissected (arrow, 200× magnification). (B) Microdissected regions of tonsil (200× magnification). (C) Determination of optimal PCR cycles for amplifying the GAPDH from microdissected tonsil. Approximately 100 cells were microdissected from human tonsil and used for RT-PCR. GAPDH-specific primers were used to amplify for 25,45 , or 65 cycles. The GAPDH product ( $476 \mathrm{bp}$ ) was the only band present at all conditions. 